\title{
Study on Synthesis and Mechanism of Melt Polymerization of L-lactic Acid
}

\author{
Ping Lan*, Jia Lv \\ Jiaxing university, Zhejiang, 314001, P. R. China
}

\begin{abstract}
A two steps direct polymerization process was developed. The first step is to produce oligomer and then the oligomer of lactic acid is polymerized with binary catalyst tin chloride dihydrate/ p-toluenesulfonic acid. In this way, the direct synthesis of poly (lactic acid)(PLLA) without any organic solvents was investigated. The properties and structures of products were characterized by nuclear magnetic resonance (NMR), FT-IR spectra, and so on. The results show that comparatively high molecular weight polymer of lactic acid can be prepared by direct processing under appropriate technological conditions and that the melt polymerization of PLLA behaviors are as the second-order reaction mechanism.
\end{abstract}

Keywords: melt polymerization, L-lactic acid, nuclear magnetic resonance (NMR), mechanism

\section{Introduction}

Polylactic acid (PLA) is expected to have wide applications not only as a biodegradable plastic but also as a biomedical material [1-4] due to its excellent properties, such as mechanical strength, transparency, safety and adjustable hydrolyzability. Main advantages of polylactic acid are good compatibility and safety. As it is degradable in the human body, it is particularly suitable for the application of implants which are used only temporarily for the healing process.

In this paper, poly(L-lactic acid) (PLLA) is prepared by direct melt polymerization instead of ring-open process (ROP) which has been already used in a great deal of literatures [3,4]. Melt polymerization is utilized to prepare a series of high Mw PLLA samples. High-resolution ${ }^{1} \mathrm{H}$ and ${ }^{13} \mathrm{C}$ NMR spectra are performed to characterize the aspolymerized copolymer and reaction mechanism is also studied.

\section{Experimental}

\subsection{Materials and Reagents}

L-lactic acid as a $90 \mathrm{wt} \%$ aqueous solution (PH90, pharmaceutical grade) was purchased from Purac Corp. (Netherland). Tin (II) chloride dihydrate, p-tolulenesulfonic acid monohydrate (TSA), chloroform, and molecular sieve ( $3 \AA$ ) were purchased from Shanghai Chemical Corp. Molecular sieve was activated at $300^{\circ} \mathrm{C}$ for 24 hours. All other reagents were analytical grade and used as received.

\subsection{Melt Polycondensation}

The first step was to make the oligomer: A given amount of L-LA aqueous solution $90 \mathrm{wt} \%$ was charged into a $100 \mathrm{~mL}$ three-necked flask. The flask was equipped with a mechanical stirrer and a reflux condenser packed with molecular sieve ( $3 \AA$ ). The reaction system was connected with a vacuum system through a cold trap. The mixture of aqueous L-LA 
was then dehydrated at a constant temperature of $150^{\circ} \mathrm{C}$ by stepwise vacuumizing the reaction system. First, the reaction system was kept at atmospheric pressure for 2 hours, then at a reduced pressure of $13,300 \mathrm{~Pa}$ for 2 hours and finally under a pressure of $1300 \mathrm{~Pa}$ for more than 4 hours. Thus viscous oligomers of L-LA were formed and the amount of water removed from the system was measured. The water byproduct and solvent was removed to obtain the oligomer. After purification, the samples were subjected to ${ }^{1} \mathrm{H}$ NMR testing, from which the degree of polymerization of the oligomer was calculated to be about 6 .

The second step was to make polymer: tin(II) chloride dihydrate corresponding to $0.4 \mathrm{wt} \%$ of the oligomer and TSA with molar ratio of tin(II)/TSA of $1 / 1$, were mixed with oligomers in the flask. Along with the pressure being reduced stepwise to reach $100 \mathrm{~Pa}$, the mixture was gradually heated to appropriate reaction temperatures (typical reaction temperature was $180^{\circ} \mathrm{C}$ ) under mechanical stirring. Then the reaction system was maintained under these conditions for predetermined hours. With the proceeding of the reaction, gradually the reaction system became viscous, and the resultant L-lactide was refluxed through the reflux condenser. At the end of the reaction, the flask was cooled down under vacuum. The obtained product was then dissolved in chloroform and subsequently precipitated into diethyl ether. Finally, the resulting solids were filtered and dried under vacuum at $65^{\circ} \mathrm{C}$.

\subsection{Polymer Characterization}

Both ${ }^{13} \mathrm{C}$ NMR and ${ }^{1} \mathrm{H}$ NMR spectra were carried out at a temperature of $300 \mathrm{~K}$ on a Bruker DMX500 NMR spectrometer. All polymer samples were dissolved in deuterated chloroform with tetramethylsilane as an internal standard. Chemical shifts in ppm were referenced relatively to chloroform at $77.00 \mathrm{ppm}$ and $7.26 \mathrm{ppm}$ in ${ }^{13} \mathrm{C}-\mathrm{NMR}$ and $1 \mathrm{H}-$
NMR spectra respectively. ${ }^{1} \mathrm{H}-\mathrm{NMR}$ spectra were acquired as $1 \%$ solution at $500 \mathrm{MHz}$. The ${ }^{13} \mathrm{C}$-NMR measurements were acquired as $10 \%$ solution at 125 $\mathrm{MHz}$, with a pulse angle of $25^{\circ}$, an acquisition time of $1.6 \mathrm{~s}$, and a delay time of $10 \mathrm{~s}$. The distribution of monomer sequence was investigated through ${ }^{13} \mathrm{C}$-NMR spectra, and monomer sequence length was derived from these data. The number-average molecular weights $(\overline{M n})$ of L-LA oligomers and polymers were determined by ${ }^{1} \mathrm{H}-\mathrm{NMR}$ spectra. In ${ }^{1} \mathrm{H}-\mathrm{NMR}$ spectra of PLLA the peaks at 5.1-5.3 ppm are due to methine protons in LA repeat units, while the peaks at $4.4 \mathrm{ppm}$ are due to methine protons in the end groups of polymers. PLLA $\overline{M n}$ can be measured by comparing the integrated area of peaks at 5.1-5.3 ppm with that at $4.4 \mathrm{ppm}$.

FT-IR spectra were recorded on a MANMNA IR-550 spectrometer (Nicolet Corp.). The samples were examined on $\mathrm{NaCl}$ discs. For each spectrum, 32 scans were taken at a resolution of $4 \mathrm{~cm}^{-1}$. All spectra presented here were expanded to full scale.

The molecular weights of PLLA were also measured by Waters 1525 HPLC. Spectrocopic-grade tetrahydrofunan (THF) was used as the mobile phase at $1 \mathrm{ml} / \mathrm{min}$. Molecular weights were calculated by the universal calibration method using polystyrene reference materials (Shodex Mw 10000, 30000, and $60000)$. The detection of the polymer fractions was performed with a Waters 2410 RI detector.

\section{Results and Discussion}

\subsection{The Characterization of PLLA}

In direct synthesis of L-LA polymers at high temperature and under vacuum, high Mn polymers could be obtained through promoting the dehydrative equilibrium to esterification direction and reducing the depolymerization of polymers to lactides. The 
typical FT-IR spectra of PLLA are given in Figure 1 The numbers of PLLA samples correspond to reaction condition.

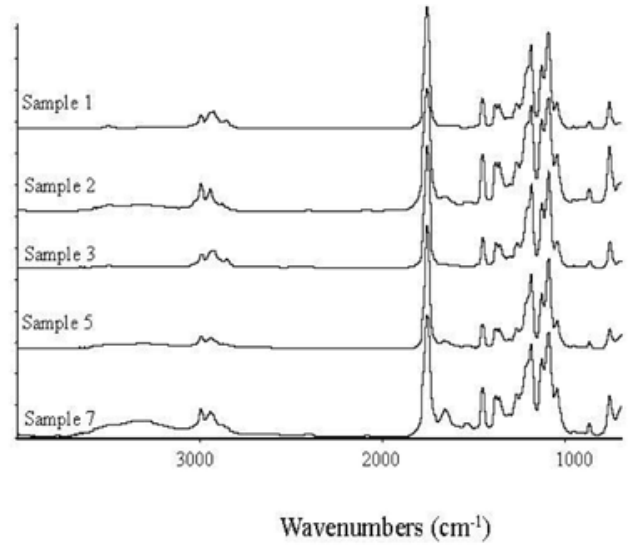

Figure 1 The FT-IR spectra of PLLA

Table I

The reaction conditions of samples for FT-IR

\begin{tabular}{lll}
\hline $\begin{array}{l}\text { Sample } \\
\text { number }\end{array}$ & \multicolumn{1}{c}{ Catalyst } & Appearance \\
\hline $1^{\text {a }}$ & Sn powder & White powder \\
2 & none & White wax \\
3 & Sn powder & Brown powder \\
5 & TSA & White wax \\
7 & Sn(II)/TSA & White powder \\
\hline
\end{tabular}

a. The sample 1 was prepared in diphenyl ether solution at $160^{\circ} \mathrm{C}$. The others were prepared at $180{ }^{\circ} \mathrm{C}$ in melt process.

All spectra of PLLA being semi-crystalline show little obvious difference from each other. Each spectrum shows a well-defined absorption at 1755 $\mathrm{cm}^{-1}$ owing to the ester carbonyl, peaks at1200$1000 \mathrm{~cm}^{-1}$ to the $\mathrm{C}-\mathrm{O}$ stretch, and peaks at 2850 $2960 \mathrm{~cm}^{-1}$ to the stretch of $\mathrm{CH}$ and $\mathrm{CH}_{3}$ groups $[5,6]$ respectively. The wide band around $3300 \mathrm{~cm}^{-1}$ due to hydrogen-bonded $\mathrm{OH}$ group denotes the existence of $\mathrm{OH}$ end group in polymer chain.

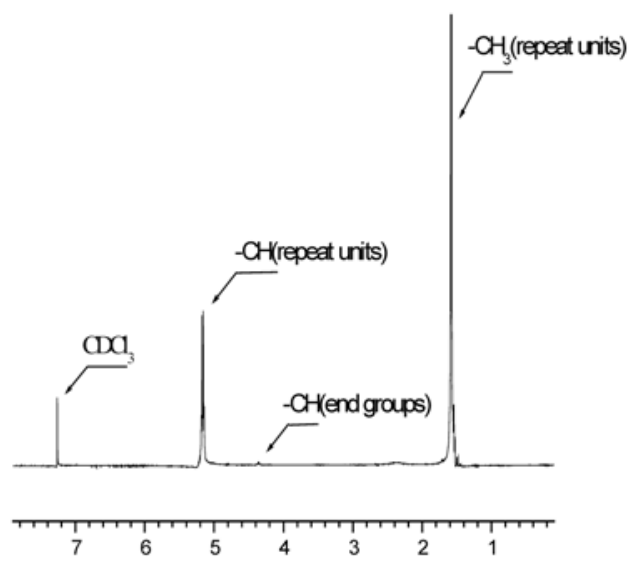

Figure 2 The typical ${ }^{1} \mathrm{H}-\mathrm{NMR}$ spectra of PLLA

The typical ${ }^{1} \mathrm{H}-\mathrm{NMR}$ and ${ }^{13} \mathrm{C}$-NMR spectra of PLLA are given in Figures 2 and 3, which show that PLLA can be prepared by direct synthesis with melt polycondensation. In ${ }^{1} \mathrm{H}$ NMR spectra of PLLA, the peaks around 5.1-5.3 ppm are due to the methine protons in LA repeat units, while the signals due to methine protons in the end groups of the polymers appear at $4.4 \mathrm{ppm}$. The peaks around $1.6 \mathrm{ppm}$ are owing to methyl protons, which often interfere with those of residual lactic acid monomer $[7,8]$. In ${ }^{13} \mathrm{C}$ - NMR spectra of PLLA, the peaks around $69 \mathrm{ppm}$ are due to the methine signals, while the signals due to the carbonyl regions appear at $169.2 \mathrm{ppm}$. The peaks around $17 \mathrm{ppm}$ are owing to methyl regions. 


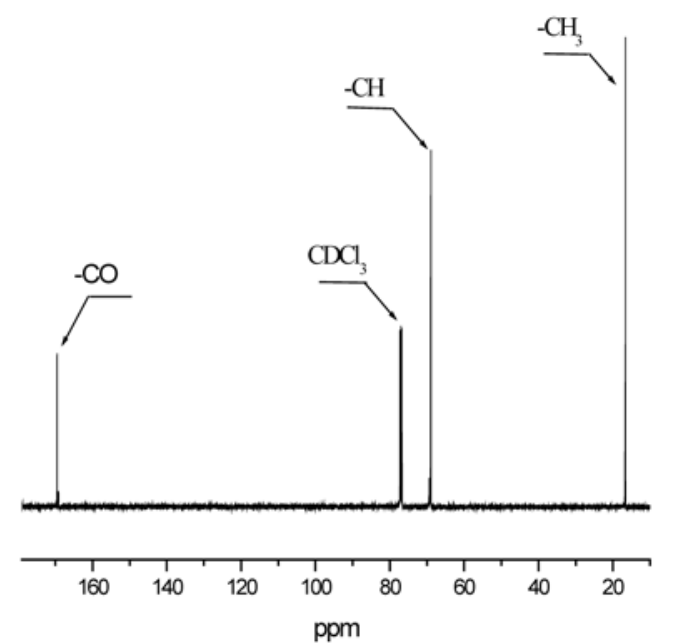

Figure3 The typical ${ }^{13} \mathrm{C}-\mathrm{NMR}$ spectra of PLLA

Table II

The ${ }^{1} \mathrm{H}-\mathrm{NMR}$ and GPC results of PLLA samples

\begin{tabular}{cccc}
\hline Sample $^{\mathrm{a}}$ & $\overline{M n} \mathrm{~b}$ & $\overline{M n} \mathrm{c}$ & $\overline{M w} \mathrm{c}$ \\
& (Daltons) & (Daltons) & (Daltons) \\
\hline $\mathrm{A}$ & 15004 & 16501 & 23199 \\
$\mathrm{~B}$ & 23178 & 25458 & 44692 \\
$\mathrm{C}$ & 31423 & 35458 & 63824
\end{tabular}

a, Sample A, B and C were prepared in a two steps direct polymerization process after different reaction time.

b, calculated from ${ }^{1} \mathrm{H}-\mathrm{NMR}$.

c, results from GPC determination.

\subsection{The mechanism and kinetics of melt polymerization of L-LA}

As for polyesters, the kinetics of polyesterification is evidently similar to that of monofunctional esterification of small-molecule condensation. The rate constant of individual polycondensation steps is essentially independent of chain length. The experimental rate expression dependencies are described in the usual manner below (equation 1). The $[\mathrm{COOH}]$ represents the diacid concenttration, and $[\mathrm{OH}]$ the diol concentration, and [Cat] the catalyst concentration. The constant k' represents the rate constant.

$$
-\frac{d[\mathrm{COOH}]}{d t}=k^{\prime}[\mathrm{Cat}][\mathrm{COOH}][\mathrm{OH}]
$$

For uncatalyzed dehydration of L-LA, the $[\mathrm{COOH}]$ and $[\mathrm{OH}]$ are present in equimolar amounts. The $\mathrm{COOH}$ groups are experimentally as a catalyst. The experimental rate expression dependencies are described as equation 2 .

$$
\begin{gathered}
-\frac{d[\mathrm{COOH}]}{d t}=k^{\prime}[\mathrm{COOH}]^{3} \\
-\frac{d[\mathrm{COOH}]}{d t}=k[\mathrm{COOH}][\mathrm{OH}]
\end{gathered}
$$

For the added acid or base is a catalyst, its apparent concentration does not change with time. Thus it need not be included in the kinetic rate expression. The reaction follows the rate expression (equation 3), where $\mathrm{k}$ in stead of k'[Cat]. In the case of polycondensation of L-lactic acid, the $[\mathrm{COOH}]$ is equal to the $[\mathrm{OH}]$. Thus the equation 4 can be introduced. Equation 4 gives on integration and substitution (equation 5), and its further arrangement gives the equation 6 . 


$$
\begin{gathered}
-\frac{d[\mathrm{COOH}]}{d t}=k[\mathrm{COOH}]^{2} \\
k t=\frac{1}{[\mathrm{COOH}]_{t}}+C_{1}=\frac{1}{[\mathrm{COOH}]_{0}(1-P)}+C_{1} \\
{[\mathrm{COOH}]_{0} k t=\frac{1}{(1-P)}+C_{2}}
\end{gathered}
$$

Here the $[\mathrm{COOH}]_{0}$ represents the $[\mathrm{COOH}]$ at the starting reaction time (i.e. $\mathrm{t}=\mathrm{t}_{0}$ ), while $[\mathrm{COOH}]$ ${ }_{t}$ represents the $[\mathrm{COOH}]$ at time $t$. The $p$ is defined as the fraction of functional groups that have reacted at time $t$, while (1-p) is the fraction of groups unreacted. Thus $[\mathrm{COOH}]_{\mathrm{t}}=[\mathrm{COOH}]_{0}(1-\mathrm{p})$. The $\mathrm{C}_{1}$ and $\mathrm{C}_{2}$ are constants. The equation 6 predicts a linear relationship of $1 /(1-p)$ with reaction time.

$$
\begin{gathered}
{\overline{D P_{N}}}_{=} \frac{[\mathrm{COOH}]_{0}}{[\mathrm{COOH}]_{t}}=\frac{1}{1-p} \\
{\overline{D P_{N}}=}_{[\mathrm{COOH}]_{0} k t+1} \\
\overline{M n}=\frac{m}{1-p}
\end{gathered}
$$

The number-average degree of polymerization $\mathrm{DP}_{\mathrm{N}}$ can be expressed as equation (7). For such second-order reactions, the relationship (equation 8) will exist at the initial reaction time. The numberaverage molecular weight $(\overline{M n})$ can be calculated from equations (9), where the $m$ is the molecular weight of the repeating unit. Considering equations (6) and (9), a linear relationship between the Mn of PLLA and reaction time will exist. Figure 4 shows the linear relationship of $\mathrm{Mn}$ with the reaction time, which means the melt polymerization of PLLA behaviors as the second-order reaction mechanism.

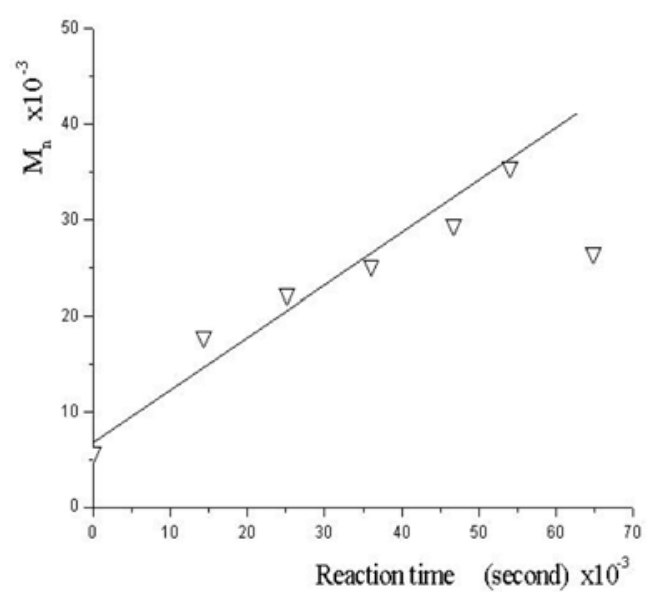

Figure 4 The linear relationship of Mn with the reaction time

The factors effecting on the obtaining of high Mn PLLA consist in both driving the dehydration equilibrium to the direction of esterification and reducing the PLLA depolymerization to lactide at high temperature and under vacuum. Even though conversion of the hydroxyl and acid groups is close to completion, any departure from the reaction stoichiometry performs a very detrimental effect on the chain length, which will cause the deviation of relationship of $\overline{M_{n}} \sim \mathrm{t}$ from linear relationship.

\section{Conclusion}

From the discussion above, the main conclusions were achieved as follows. Comparatively high molecular weight polymer of lactic acid can be prepared by direct processing under appropriate technological conditions and that the melt polymerization of PLLA behaviors as the second-order reaction mechanism. 


\section{Acknowledgement}

The authors thank the Science and Technology Project of Jiaxing City, Zhejiang(2007BY5007) for the financial support.

\section{References:}

[1] Maria L. Ehrenfried. The effect of tri-calcium phosphate (TCP) addition on the degradation of polylactide-co-glycolide (PLGA). J Mater Sci-Mater M 2008;19:459-466.

[2] Hongyan T. Preparation, characterization and mechanical properties of the polylactide/perlite and the polylactide/montmorillonite composites. J Mater Sci 2007;42:3244-3250.

[3] Chen G, Ushida T, Tateishi T. Preparation of poly(Llactic acid) and poly(DL-lactic-co-glycolic acid) foams by use of ice microparticulates. Biomaterials 2001;25:2563-2567.
[4] Solarski S. (Plasticized) Polylactide/clay nanocomposite textile: thermal, mechanical, shrinkage and fire properties. J Mater Sci 2007;42:5105-5117.

[5] Miyata T, Masuko T. Crystallization behaviour of poly(L-lactide). Polymer 1998;39:5515-5521

[6] Wang N, Wu XS, Lujian-Upton H, Donahue E, Siddiqui A. Synthesis and characterization of lactic/ glycolic acid oligomers. J Polym Mater Sci Eng 1997;76:373-374

[7] Esoartero JL, Rashkov I, Li SM, Manolova N, Vert M. NMR analysis of low molecular weight poly(lactic acid)s. Macromolecules 1996;29:35353539.

[8] Choi EJ, Park JK, Chang HN. Effect of polymerization catalysts on the microstructure of poly(L-LA-co- $\varepsilon C L)$. J Polym Sci Polym Phys 1994;32:2481-2489. 